\title{
Quasiconvex constrained multicriteria continuous location problems: Structure of nondominated solution sets
}

\author{
J. Puerto ${ }^{\mathrm{a}, *}$, A.M. Rodríguez-Chía ${ }^{\mathrm{b}}$ \\ ${ }^{a}$ Facultad de Matemáticas, Univ. de Sevilla, C/Tarfia s/n, 41012 Sevilla, Spain \\ ${ }^{\mathrm{b}}$ Facultad de Ciencias, Universidad de Cádiz, Pol. Río San Pedro, 11510 Puerto Real (Cádiz), Spain
}

Available online 7 July 2006

\begin{abstract}
In this paper, we consider constrained multicriteria continuous location problems in two-dimensional spaces. In the literature, the continuous multicriteria location problem in two-dimensional spaces has received special attention in the last years, although only particular instances of convex functions have been considered. Our approach only requires the functions to be strictly quasiconvex and inf-compact. We obtain a geometrical description that provides a unified approach to handle multicriteria location models in two-dimensional spaces which has been implemented in MATHEMATICA.
\end{abstract}

(C) 2006 Elsevier Ltd. All rights reserved.

Keywords: Location; Multicriteria; Geometrical description

\section{Introduction}

Considering uncertainty in Operations Research models is a core problem nowadays. A number of problems that we find in the real-world present elements which escape the control of the decision maker. The uncertainty is often originated by the impossibility of choosing only one scenario where the problem is likely to occur or because it is unclear which objective function should be optimized. In both cases, the necessity of finding "good" solutions for different criteria (scenarios), rather than for only one can be addressed considering multicriteria problems.

The constrained multicriteria location problem in a space $X$ can be written, in great generality, as

$$
v-\min _{y \in Y}\left(F_{1}(y), \ldots, F_{k}(y)\right),
$$

where $v-$ min stands for vector minimization, $F_{1}(\cdot), \ldots, F_{k}(\cdot): X \rightarrow \mathbb{R}$ represent the different criteria and $Y \subseteq X$ the feasible region.

A well-known multicriteria model in Location Theory is the Point-Objective location problem. It can be considered as a particular instance of the above formulation where each function $F_{i}(\cdot)=\gamma\left(\cdot-a_{i}\right)$, being $A=\left\{a_{1}, \ldots, a_{k}\right\}$ the set of demand facilities of a location problem, and $\gamma\left(\cdot-a_{i}\right)$ the function that measures the distances to $a_{i}$. The unconstrained version of this problem, $Y=X=\mathbb{R}^{2}$, was dealt firstly in [1] for the $l_{2}$-norm. Later, [2] solved this problem for polyhedral gauges. Other references devoted to study modifications of the Point-Objective location models are [3-13],

\footnotetext{
* Corresponding author. Tel.: +34 95 4557940; fax: +34954622800.

E-mail addresses: puerto@us.es (J. Puerto), antonio.rodriguezchia@uca.es (A.M. Rodríguez-Chía).
} 
among others. It is worth noting that from the above references only [3-8,11] consider the constrained case. Moreover, these references study the particular case of the Point-Objective location problem where distances are measured with the same norm. Only [6,7] analyze the constrained Point-Objective location problem with different norms but their goal is to characterize solutions in location problems with voting criteria.

The unconstrained version of the multicriteria problem being $F_{i}(\cdot)$, for $i=1, \ldots, k$, weighted sums of the distances measured with the $l_{1}$-norm (Weber problem with the Manhattan norm) in $\mathbb{R}^{2}$, was solved by Hamacher and Nickel [14]. The problem where the functions, $F_{i}(\cdot)$ for $i=1, \ldots, k$, are weighted sums of distances measured with any norm was studied by Puerto and Fernández [15,16]. In addition, [17] considered a quadratic bicriteria location model. Again, the reader can see that these references only deal with the unconstrained case.

The multicriteria location problem with regional demand and objective functions $F_{i}(\cdot)$, for $i=1, \ldots, k$, being inf-distance functions (recall that inf-distance means distance to the closest point of each demand set) was solved by Rodríguez-Chía [18]. Refs. [19,20,29] give a geometrical characterization of a general multicriteria problem where the objective functions are only restricted to be convex. In order to do that the authors reduce the original problem to resolve multicriteria subproblems involving a lower number of objective functions. However, this characterization does not provide a geometrical construction of the nondominated solutions for constrained multicriteria problems.

Scanning the literature, we can see that the multicriteria location problem has received special attention in the last years, (see [21, Chapter 19] for a survey on multicriteria location problems). However, there is a lack of a common geometrical description of the nondominated solution set for the constrained version of these problems. In this paper, we develop a unified approach to solve constrained continuous multicriteria location problems in two-dimensional spaces with strictly quasiconvex inf-compact functions. The novelty of the results in this paper, within the location analysis field, is to provide a methodology to handle multicriteria location problems with different norms at the different demand points. Despite of the generality of the considered problem, the results obtained in this paper are easy to understand and the proofs basically rely on Convex Analysis tools. Our results are not only at the theoretical level. Instead, they allow to actually construct the complete solution set. In this regard, we also provide examples that illustrate the results, and we relate these results with the existing ones, showing that our characterization unifies different known results in the literature.

The paper is organized in five sections. In the second section we present the model and the notation used throughout the paper. In Section 3, we give a characterization of the nondominated solution set of the bicriteria constrained problem. In Section 4, we analyze the three-criteria and the general $k$-criteria problem. For the sake of readability, the proofs of several technical lemmas are deferred to the Appendix. The Appendix also includes the implementation of our results for the bicriteria problem, as well as details of the resolution of the examples using this code. Finally, Section 5 is devoted to the concluding remarks.

\section{The model}

We consider $F_{1}, \ldots, F_{k}: \mathbb{R}^{2} \longrightarrow \mathbb{R}$ a finite set of strictly quasiconvex, inf-compact functions which represent different criteria or scenarios, and a closed, convex, feasible region $Y \subseteq \mathbb{R}^{2}$ (see [4] for further analysis with nonconvex feasible regions). Recall that a real function $f(\cdot)$ is said to be inf-compact if its lower level sets $\{x: f(x) \leqslant \rho\}$ are compact for any $\rho \in \mathbb{R}$, notice that a lower semicontinuous function bounded on each compact set is inf-compact. Our goal is to find the set of points $y \in Y$ such that there is no $z \in Y$ that improves the value of $F_{i}(y)$ for all $i=1, \ldots, k$. This problem can be formulated as finding the set of weakly efficient solutions to the following vector minimization problem:

$$
v-\min _{y \in Y}\left(F_{1}(y), \ldots, F_{k}(y)\right)
$$

We let $\mathrm{WE}\left(F_{1}, \ldots, F_{k} ; Y\right)$ denote the nondominated solution set for this problem defined by

$$
\mathrm{WE}\left(F_{1}, \ldots, F_{k} ; Y\right):=\left\{y \in Y: \forall z(\neq y) \in Y: \exists F_{i} \text { for some } i=1, \ldots, k \text { with } F_{i}(y) \leqslant F_{i}(z)\right\}
$$

In the case $Y=\mathbb{R}^{2}$ (unconstrained case) we drop the reference to the constraint and therefore it is denoted by $\mathrm{WE}\left(F_{1}, \ldots, F_{k}\right)$. Usually, this set is called the set of weakly efficient points. 
In order to improve the readability, we include below some concepts and the notation used throughout the paper. The lower level set of the function $F(\cdot)$ for a value $\rho \in \mathbb{R}$ is the set $L \leqslant(F, \rho):=\left\{x \in \mathbb{R}^{2}: F(x) \leqslant \rho\right\}$, the strict lower level set is $L_{<}(F, \rho):=\left\{x \in \mathbb{R}^{2}: F(x)<\rho\right\}$, and the level set $L_{=}(F, \rho):=\left\{x \in \mathbb{R}^{2}: F(x)=\rho\right\}$.

For a strictly quasiconvex, inf-compact function $F_{i}(\cdot)$ we will use the notation

$$
\mathscr{X}^{*}\left(F_{i}\right):=\arg \min _{x \in \mathbb{R}^{2}} F_{i}(x)
$$

to refer to its set of minimizers. It should be noted that this set is closed and convex.

For two functions, $F_{i}(\cdot)$ and $F_{j}(\cdot)$, with $i, j \in\{1, \ldots, k\}$, let

$$
\begin{aligned}
& I_{i j}^{\leqslant}(x):=L_{\leqslant}\left(F_{i}, F_{i}(x)\right) \cap L_{\leqslant}\left(F_{j}, F_{j}(x)\right), \\
& I_{i j}^{<}(x):=L_{<}\left(F_{i}, F_{i}(x)\right) \cap L_{<}\left(F_{j}, F_{j}(x)\right), \\
& I_{i j}^{=}(x):=L_{=}\left(F_{i}, F_{i}(x)\right) \cap L_{=}\left(F_{j}, F_{j}(x)\right) .
\end{aligned}
$$

The tangent cone $T_{B}(x)$ to the set $B$ at point $x$ is

$$
T_{B}(x):=\operatorname{cl}(\text { cone }(B-x)),
$$

where for any set $S, \operatorname{cl}(S)$ stands for the topological closure of $S$. In addition, for a general closed set $A \subset \mathbb{R}^{2}$ we denote by $\partial(A)$ the boundary of this set, $\operatorname{ri}(A)$ its relative interior and $\operatorname{int}(A)$ its interior. For two points $x$ and $y$, we let $\overline{x y}$ denote the segment defined by $x$ and $y$. Finally, for a strictly quasiconvex, inf-compact function we define the projection of $F(\cdot)$ onto the closed set $A$ as

$$
\underset{A}{\operatorname{proj}}(F)=\arg \min _{a \in A} F(a) .
$$

In the next sections we obtain a geometrical characterization of the nondominated solutions for Problem (1). Before that, we give a basic result stating a necessary and sufficient condition for a point to be weakly efficient. A similar result can be found in [22,23], therefore we omit its proof.

\section{Theorem 2.1. For a point $y \in Y$, the following conditions are equivalent:}

1. $y \in \mathrm{WE}\left(F_{1}, \ldots, F_{k} ; Y\right)$,

2. $\bigcap_{i=1}^{k} L_{<}\left(F_{i}, F_{i}(y)\right) \cap Y=\emptyset$.

Notice that for $k=2$ and $Y=\mathbb{R}^{2}$, this result provides a geometrical characterization of $\operatorname{WE}\left(F_{1}, \ldots, F_{k} ; Y\right)$ as the set of tangent points between $L_{=}\left(F_{1}, \rho\right)$ and $L_{=}\left(F_{2}, \rho^{\prime}\right)$ for $\rho, \rho^{\prime} \in \mathbb{R}$.

In what follows, we will analyze the problem depending on the number of functions and on the relative position between the solution set of the unconstrained problem and the feasible region.

\section{The bicriteria problem}

We start studying in this section a simpler version of Problem (1) with only two objective functions. We will show later in Section 4 that the essential building blocks in obtaining the solution set $\mathrm{WE}\left(F_{1}, \ldots, F_{k} ; Y\right)$ are the simpler sets $\operatorname{WE}\left(F_{i}, F_{j} ; Y\right)$ for all $i, j$. For this reason, we concentrate in this section on the bicriteria case. Moreover, due to its importance in building examples, we have developed an implementation in MATHEMATICA (see Appendix) that illustrates the usefulness of the results.

To analyze the problem we distinguish two cases. The intersection of $\operatorname{WE}\left(F_{1}, F_{2}\right)$ and the feasible region $Y$ is empty or it is nonempty.

\subsection{The case $\mathrm{WE}\left(F_{1}, F_{2}\right) \cap Y=\emptyset$}

In this subsection we obtain a geometrical characterization of $\operatorname{WE}\left(F_{1}, F_{2} ; Y\right)$ when $\operatorname{WE}\left(F_{1}, F_{2}\right) \cap Y=\emptyset$. Therefore, we assume in the following that $\operatorname{WE}\left(F_{1}, F_{2}\right) \cap Y=\emptyset$. 
Definition 3.1. The set $\partial\left(\operatorname{proj}_{Y}\left(F_{1}\right), \operatorname{proj}_{Y}\left(F_{2}\right)\right)$ is the connected path on the boundary of $Y$ that satisfies:

(i) $\operatorname{proj}_{Y}\left(F_{1}\right) \cup \operatorname{proj}_{Y}\left(F_{2}\right) \subseteq \partial\left(\operatorname{proj}_{Y}\left(F_{1}\right), \operatorname{proj}_{Y}\left(F_{2}\right)\right)$.

(ii) $\partial\left(\operatorname{proj}_{Y}\left(F_{1}\right), \operatorname{proj}_{Y}\left(F_{2}\right)\right) \backslash\left(\operatorname{proj}_{Y}\left(F_{1}\right) \cup \operatorname{proj}_{Y}\left(F_{2}\right)\right)$ is a connected set.

(iii) Let $C:=\operatorname{WE}\left(F_{1}, F_{2}\right) \cup \overline{x_{1}^{*} \bar{y}_{1}} \cup \overline{\bar{y}_{1} \bar{y}_{2}} \cup \overline{x_{2}^{*} \bar{y}_{2}}$, with $\bar{y}_{i} \in \operatorname{proj}_{Y}\left(F_{i}\right), x_{i}^{*} \in \mathscr{X}^{*}\left(F_{i}\right)$ for $i=1,2$ and let encl $(C)$ be the bounded region encircled by $C$ (notice that $C \cap \operatorname{encl}(C)=\emptyset)$.

(a) If $\partial(Y) \cap \operatorname{encl}(C)=\emptyset$ then $\overline{\bar{y}}_{1} \bar{y}_{2} \subseteq \partial\left(\operatorname{proj}_{Y}\left(F_{1}\right), \operatorname{proj}_{Y}\left(F_{2}\right)\right)$.

(b) If $\partial(Y) \cap \operatorname{encl}(C) \neq \emptyset$ then $\partial\left(\operatorname{proj}_{Y}\left(F_{1}\right), \operatorname{proj}_{Y}\left(F_{2}\right)\right) \backslash\left(\operatorname{proj}_{Y}\left(F_{1}\right) \cup \operatorname{proj}_{Y}\left(F_{2}\right)\right) \subseteq \operatorname{encl}(C)$.

Recall that given two points $x$ and $y$, we let $\overline{x y}$ denote the segment joining these two points. By Lemma A.1 in the Appendix, we have that this definition is well-stated. The following technical lemma the proof of which can be found in the Appendix is needed to prove the main result of this subsection.

Lemma 3.1. Let $y_{1}, y_{2}\left(y_{1} \neq y_{2}\right) \in \partial(Y) \backslash\left(\operatorname{proj}_{Y}\left(F_{1}\right) \cup \operatorname{proj}_{Y}\left(F_{2}\right)\right)$ such that $F_{1}\left(y_{1}\right)=F_{1}\left(y_{2}\right), F_{2}\left(y_{1}\right) \leqslant F_{2}\left(y_{2}\right)$ and $L \leqslant\left(F_{1}, F_{1}\left(y_{1}\right)\right) \cap \operatorname{proj}_{Y}\left(F_{2}\right)=\emptyset$. If $\mathrm{WE}\left(F_{1}, F_{2}\right) \cap Y=\emptyset$ then

(i) $y_{2} \notin \mathrm{WE}\left(F_{1}, F_{2} ; Y\right)$.

(ii) $y_{2} \notin \partial\left(\operatorname{proj}_{Y}\left(F_{1}\right), \operatorname{proj}_{Y}\left(F_{2}\right)\right)$.

The main result in this subsection states that the structure of $\mathrm{WE}\left(F_{1}, F_{2} ; Y\right)$ is described as a part of the boundary of $Y$ delimited by the minimizers of $F_{1}$ and $F_{2}$ on $Y$.

Theorem 3.1. If $\mathrm{WE}\left(F_{1}, F_{2}\right) \cap Y=\emptyset$, we have that

$$
\mathrm{WE}\left(F_{1}, F_{2} ; Y\right)=\partial\left(\operatorname{proj}_{Y}\left(F_{1}\right), \operatorname{proj}_{Y}\left(F_{2}\right)\right) \text {. }
$$

Remark 3.1. This result unifies for two-dimensional spaces previous characterizations in the literature. Taking $F_{i}(x)=$ $\left\|x-a_{i}\right\|$ with $a_{i} \in \mathbb{R}^{2}$ for $i=1, \ldots, n$, when $\|\cdot\|$ is the Euclidean norm we get Theorem 2 in [3], where the constrained solution set is the orthogonal projection of the convex hull of $a_{i} \in \mathbb{R}^{2}$, for $i=1, \ldots, n$. When $\|\cdot\|$ is a strictly convex norm (the same with respect to the different existing facilities $a_{i}, i=1, \ldots, n$ ) we get Theorem 1 in [8], where the constrained solution set is the projection of the convex hull of the existing facilities using this norm. When $\|\cdot\|$ is an arbitrary norm (but the same with respect to the different existing facilities $a_{i}, i=1, \ldots, n$ ) we get Theorem 4.2 in [11]. There, the constrained solution set is the projection of the unconstrained solution set. It should be noted that these papers use the concept of projection with regard to the unique norm that appears in their objective functions. However, in our model it is not possible to project the unconstrained solution set because different norms (functions) are simultaneously considered. In Example 3.2, we illustrate how to obtain these characterizations applying Theorem 3.1.

Proof. First of all, we prove that $\operatorname{WE}\left(F_{1}, F_{2} ; Y\right) \cap \operatorname{int}(Y)=\emptyset$. In order to do that, assume that there exists $y \in$ $\mathrm{WE}\left(F_{1}, F_{2} ; Y\right) \cap \operatorname{int}(Y)$. Since $\mathrm{WE}\left(F_{1}, F_{2}\right) \cap Y=\emptyset$ we have that $y \notin \mathrm{WE}\left(F_{1}, F_{2}\right)$; and it implies that $I_{12}^{<}(y) \neq \emptyset$. Besides, using that $y \in \operatorname{int}(Y)$ and the convexity of the level sets, we have that $I_{12}^{<}(y) \cap Y \neq \emptyset$. Thus, Theorem 2.1 states that $y \notin \mathrm{WE}\left(F_{1}, F_{2} ; Y\right)$. Hence, $\mathrm{WE}\left(F_{1}, F_{2} ; Y\right) \subseteq \partial(Y)$.

On the other hand, we prove that $\operatorname{proj}_{Y}\left(F_{1}\right) \cup \operatorname{proj}_{Y}\left(F_{2}\right) \subseteq \mathrm{WE}\left(F_{1}, F_{2} ; Y\right)$. This inclusion holds because there exists no point $z \in Y$ such that $F_{i}(z)<F_{i}(y) \forall y \in \operatorname{proj}_{Y}\left(F_{i}\right)$ for $i=1,2$.

If it holds that $\operatorname{proj}_{Y}\left(F_{1}\right) \cap \operatorname{proj}_{Y}\left(F_{2}\right) \neq \emptyset$ then $\operatorname{WE}\left(F_{1}, F_{2} ; Y\right)=\operatorname{proj}_{Y}\left(F_{1}\right) \cup \operatorname{proj}_{Y}\left(F_{2}\right)$ because any point of $Y \backslash\left(\operatorname{proj}_{Y}\left(F_{1}\right) \cup \operatorname{proj}_{Y}\left(F_{2}\right)\right)$ is dominated by any point of $\left(\operatorname{proj}_{Y}\left(F_{1}\right) \cap \operatorname{proj}_{Y}\left(F_{2}\right)\right)$. In this case, the result is proved because $\partial\left(\operatorname{proj}_{Y}\left(F_{1}\right), \operatorname{proj}_{Y}\left(F_{2}\right)\right)=\operatorname{proj}_{Y}\left(F_{1}\right) \cup \operatorname{proj}_{Y}\left(F_{2}\right)$.

In what follows we assume that $\operatorname{proj}_{Y}\left(F_{1}\right) \cap \operatorname{proj}_{Y}\left(F_{2}\right)=\emptyset$. Now, we prove that there are points on one side of the boundary of $Y$ in a neighborhood of $\operatorname{proj}_{Y}\left(F_{1}\right)$ which do not belong to $\operatorname{WE}\left(F_{1}, F_{2} ; Y\right)$. Let $\bar{y}_{1} \in \operatorname{proj}_{Y}\left(F_{1}\right)$ and $\varepsilon>0$ be small enough such that $L \leqslant\left(F_{1}, F_{1}\left(\bar{y}_{1}\right)+\varepsilon\right) \cap \operatorname{proj}_{Y}\left(F_{2}\right)=\emptyset$. Since $\operatorname{proj}_{Y}\left(F_{1}\right) \subset L \leqslant\left(F_{1}, F_{1}\left(\bar{y}_{1}\right)+\varepsilon\right) \cap \partial(Y)$ there exist two points $y_{1}, y_{2}\left(y_{1} \neq y_{2}\right) \in L_{=}\left(F_{1}, F_{1}\left(\bar{y}_{1}\right)+\varepsilon\right) \cap \partial(Y)$ such that $y_{1}$ is separated from $y_{2}$ by $\operatorname{proj}_{Y}\left(F_{1}\right)$ in at least one of the two paths on $\partial(Y)$ that join $y_{1}$ and $y_{2}$. (Without loss of generality we assume that $F_{2}\left(y_{1}\right) \leqslant F_{2}\left(y_{2}\right)$.) By Lemma 3.1 we have that $y_{2} \notin \mathrm{WE}\left(F_{1}, F_{2} ; Y\right)$ and $y_{2} \notin \partial\left(\operatorname{proj}_{Y}\left(F_{1}\right), \operatorname{proj}_{Y}\left(F_{2}\right)\right)$. 


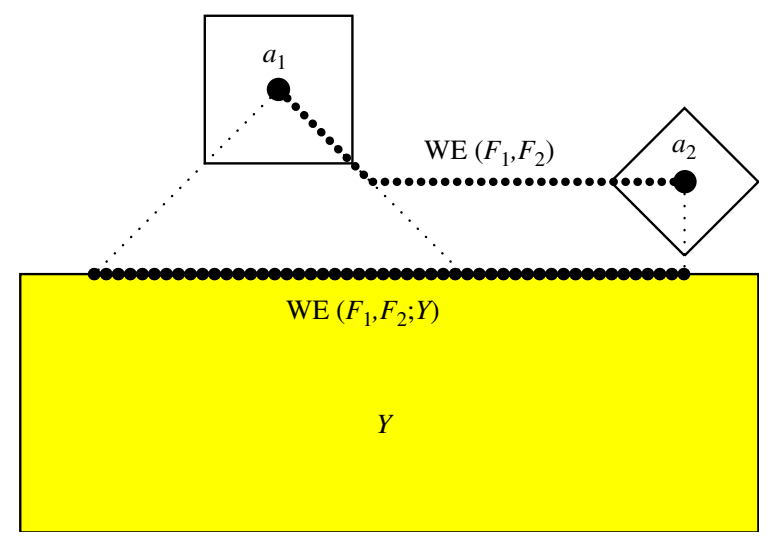

Fig. 1. Illustration of Example 3.1.

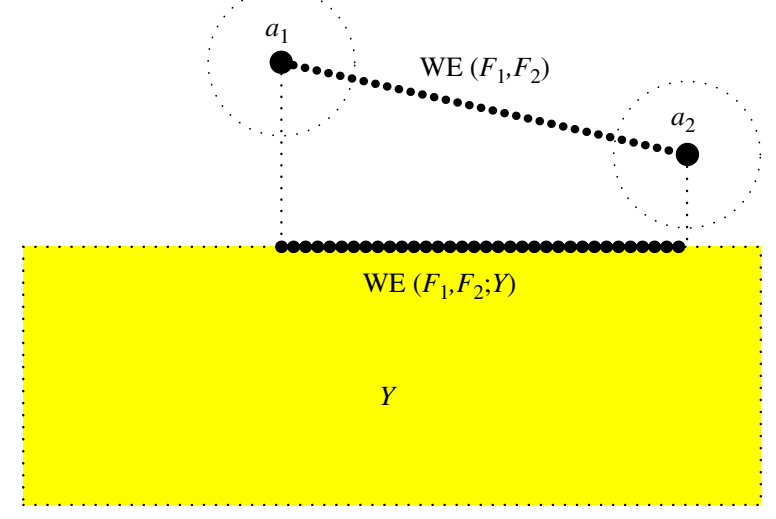

Fig. 2. Illustration of Example 3.2.

Notice that the same result can be obtained in a neighborhood of the appropriate side of $\operatorname{proj}_{Y}\left(F_{2}\right)$.

Hence, we have proved that there exist points of $\partial(Y)$ in a neighborhood of $\operatorname{proj}_{Y}\left(F_{1}\right)$ and $\operatorname{proj}_{Y}\left(F_{2}\right)$ that do not belong to $\mathrm{WE}\left(F_{1}, F_{2} ; Y\right)$ nor to $\partial\left(\operatorname{proj}_{Y}\left(F_{1}\right), \operatorname{proj}_{Y}\left(F_{2}\right)\right)$. Therefore, using the connectedness property of the solution set (see [24]) and that $\mathrm{WE}\left(F_{1}, F_{2} ; Y\right) \subseteq \partial(Y)$ we obtain that $\mathrm{WE}\left(F_{1}, F_{2} ; Y\right)=\partial\left(\operatorname{proj}_{Y}\left(F_{1}\right), \operatorname{proj}_{Y}\left(F_{2}\right)\right)$.

Example 3.1. Let $a_{1}=(7,5), a_{2}=(18,3)$ and let the feasible region, $Y$, be defined by the convex hull of the points $\{(0,0),(20,0),(20,-7),(0,-7)\}$. Consider the functions $F_{1}(x)=\left\|x-a_{1}\right\|_{\infty}$ and $F_{2}(x)=\left\|x-a_{2}\right\|_{1}$. Our goal is to find the solution set $\operatorname{WE}\left(F_{1}, F_{2} ; Y\right)$. By Theorem 3.1, this set is determined by the path on the boundary of $Y$ that connects the projections of both functions onto $Y$, see Fig. 1. The solution set is depicted as the path of boundary of $Y$ drawn in thick line.

Example 3.2. Let $a_{1}=(7,5), a_{2}=(18,3)$ and let the feasible region, $Y$, be defined by the convex hull of the points $\{(0,0),(20,0),(20,-7),(0,-7)\}$. Consider the functions $F_{1}(x)=\left\|x-a_{1}\right\|_{2}$ and $F_{2}(x)=\left\|x-a_{2}\right\|_{2}$. Our goal is to find the solution set $\operatorname{WE}\left(F_{1}, F_{2} ; Y\right)$. By Theorem 3.1, this set is determined by the path on the boundary of $Y$ that connects the projections of both functions onto $Y$, see Fig. 2. The solution set is depicted as the path on the boundary of $Y$ drawn in thick line. Notice that this set is the projection, with the $l_{2}$-norm, of the convex hull of $a_{1}$ and $a_{2}$ or equivalently, the projection of the solution set for the unconstrained problem. 


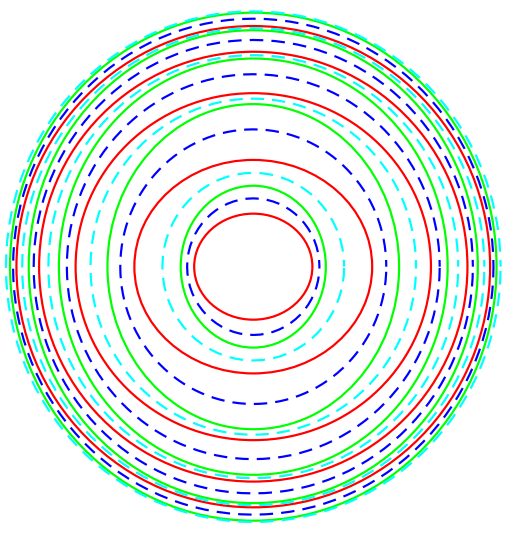

(a)

Level sets

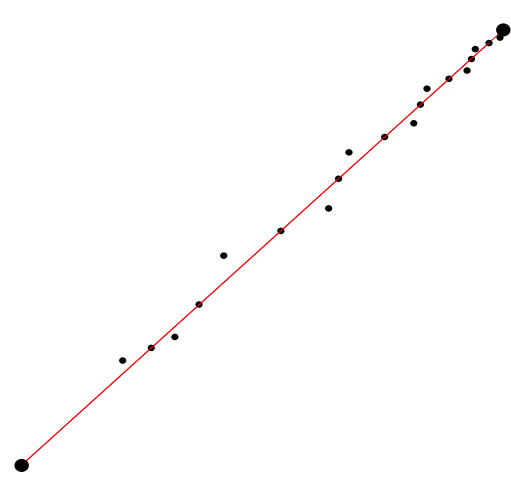

(b)

Tangent points

Fig. 3. Illustration of Example 3.3.

\subsection{The case $\operatorname{WE}\left(F_{1}, F_{2}\right) \cap Y \neq \varnothing$}

In this subsection, we analyze the case where $\operatorname{WE}\left(F_{1}, F_{2}\right) \cap Y$ is nonempty. In this case, if $\operatorname{proj}_{Y}\left(F_{1}\right) \cap \operatorname{proj}_{Y}\left(F_{2}\right) \neq \varnothing$ we have that the points in $Y \backslash\left(\operatorname{proj}_{Y}\left(F_{1}\right) \cup \operatorname{proj}_{Y}\left(F_{2}\right)\right)$ are dominated by the points of $\operatorname{proj}_{Y}\left(F_{1}\right) \cap \operatorname{proj}_{Y}\left(F_{2}\right)$. Thus, we have that $\mathrm{WE}\left(F_{1}, F_{2} ; Y\right)=\operatorname{proj}_{Y}\left(F_{1}\right) \cup \operatorname{proj}_{Y}\left(F_{2}\right)$. Therefore, in the following we assume that $\operatorname{proj}_{Y}\left(F_{1}\right) \cap \operatorname{proj}_{Y}\left(F_{2}\right)=\emptyset$.

Now we compute the set of nondominated points when that intersection is empty. Since $\partial(Y), \operatorname{proj}_{Y}\left(F_{1}\right), \operatorname{proj}_{Y}\left(F_{2}\right)$ and $\operatorname{WE}\left(F_{1}, F_{2}\right)$ are closed connected sets, we have that the set $\partial(Y) \backslash\left(\mathrm{WE}\left(F_{1}, F_{2}\right) \cup \operatorname{proj}_{Y}\left(F_{1}\right) \cup \operatorname{proj}_{Y}\left(F_{2}\right)\right)$ consists of open paths on the boundary of $Y$. (Notice that by the connectedness of the weakly efficient set, the number of open paths mentioned above is denumerable.) Let $H_{j}$ with $j \in J$ denote these open paths enumerated counterclockwise along the boundary of $Y$, starting from the projection of $F_{1}(\cdot)$. For any $H_{j}$, we denote the extreme points of its closure by $h_{j_{1}}$ and $h_{j_{2}}$. Notice that $h_{j_{1}}, h_{j_{2}} \in \mathrm{WE}\left(F_{1}, F_{2}\right) \cup \operatorname{proj}_{Y}\left(F_{1}\right) \cup \operatorname{proj}_{Y}\left(F_{2}\right)$. In what follows, we assume without loss of generality that: (1) $\operatorname{proj}_{Y}\left(F_{i}\right) \cap \operatorname{int}(Y)=\emptyset$ for $i=1,2$; and (2) $F_{1}\left(h_{j_{1}}\right)<F_{1}\left(h_{j_{2}}\right), F_{2}\left(h_{j_{2}}\right)<F_{2}\left(h_{j_{1}}\right)$ for all $j \in J$. (see Remark A.1)

The next example proves that we can have infinitely many elements in $J$. In fact, we show two strictly quasiconvex, inf-compact functions, $F_{1}$ and $F_{2}$, such that, $W E\left(F_{1}, F_{2}\right)$ intersected with a linear feasible region has an infinite, yet denumerable number of isolated intersection points.

Example 3.3. Let $F_{1}(x)=\|x-(11,11)\|_{1}$ and let $F_{2}$ be defined by its level curves. To define the level sets of $F_{2}$ we use a parametrization of the level value $\rho \in[0,11]$ (see Fig. 3(a)). The level set $L \leqslant\left(F_{2}, \rho\right)$ must satisfy:

1. $L \leqslant\left(F_{2}, \rho\right)$ is a closed, convex set.

2. $L_{=}\left(F_{2}, \rho\right)$ is a smooth curve.

3. $L \leqslant\left(F_{2}, \rho\right) \subset L \leqslant\left(F_{2}, \rho^{\prime}\right)$ for all $\rho<\rho^{\prime}$ and $L_{=}\left(F_{2}, 0\right)=\{(0,0)\}$.

4. If $\rho=\rho_{n, i}, i=1, \ldots, 4, n \in \mathbb{N}$, then

$$
\begin{aligned}
& L_{=}\left(F_{2}, \rho_{n, 1}:=\sum_{k=0}^{5 n} 1.1^{-k}\right)=\left\{\left(x_{1}, x_{2}\right): \frac{x_{1}^{2}}{\left(\rho_{n, 1}\right)^{2}}+\frac{x_{2}^{2}}{\left(\rho_{n, 1}-1.1^{-5 n}\right)^{2}}=1\right\}, \\
& L_{=}\left(F_{2}, \rho_{n, 2}:=\sum_{k=0}^{5 n+1} 1.1^{-k}\right)=\left\{\left(x_{1}, x_{2}\right): \frac{x_{1}^{2}}{\left(\rho_{n, 2}\right)^{2}}+\frac{x_{2}^{2}}{\left(\rho_{n, 2}\right)^{2}}=1\right\},
\end{aligned}
$$




$$
\begin{aligned}
& L=\left(F_{2}, \rho_{n, 3}:=\sum_{k=0}^{5 n+2} 1.1^{-k}\right)=\left\{\left(x_{1}, x_{2}\right): \frac{x_{1}^{2}}{\left(\rho_{n, 3}\right)^{2}}+\frac{x_{2}^{2}}{\left(\rho_{n, 3}+1.1^{-(5 n+3)}\right)^{2}}=1\right\}, \\
& L=\left(F_{2}, \rho_{n, 4}:=\sum_{k=0}^{5 n+3} 1.1^{-k}+1.2^{-(5 n+4)}\right)=\left\{\left(x_{1}, x_{2}\right): \frac{x_{1}^{2}}{\left(\rho_{n, 4}\right)^{2}}+\frac{x_{2}^{2}}{\left(\rho_{n, 4}\right)^{2}}=1\right\} .
\end{aligned}
$$

Notice that for each $n \geqslant 1$, with the values $\rho_{n, i}, i=1, \ldots, 4$, we have defined four level curves: two ellipses and two circumferences, such that, ellipse (4) is included in the region delimited by circumference (5), circumference (5) is included in the region delimited by ellipse (6) and ellipse (6) is included in the region delimited by circumference (7). Moreover, we have that $L_{\leqslant}\left(F_{2}, \rho_{n, 4}\right) \subseteq L_{\leqslant}\left(F_{2}, \rho_{n+1,1}\right)$ for $n \geqslant 1$, see Fig. 3(a).

Since $F_{2}$ is constructed to be strictly quasiconvex, we have that $\mathrm{WE}\left(F_{1}, F_{2}\right)$ is a connected curve joining $(0,0)$ and $(11,11)$ consisting of the consecutive tangent points between the level sets of $F_{1}$ and $F_{2}$. We can see that the tangent points between the level sets of $F_{1}$ and those of $F_{2}$ defined by (4), (5), (6) and (7) are represented in Fig. 3(b). For each two tangent points included in the segment $\overline{(0,0)(11,11)}$ (tangent points corresponding to the level sets of type (5) and (7)) there are alternatively one tangent point above the segment and another below the segment (tangent points corresponding to the level sets of type (4) and (6)).

The level curves of $F_{2}$ accumulate onto the circumference centered at $(0,0)$ and radius 11 . Then, since the weakly efficient set is connected there must exist infinitely many intersections of $\operatorname{WE}\left(F_{1}, F_{2}\right)$ with the segment defined by $(0,0)$ and $(11,11)$.

The reader may notice that this example is not just a theoretical construction. In fact, the code in MATHEMATICA that realizes the example is described in Implementation A. 2 at the end of the Appendix.

Before obtaining the characterization result of the solution set for two functions, we give two technical lemmas which proofs can be seen in the Appendix.

\section{Lemma 3.2. It holds that}

$$
L \leqslant\left(F_{i}, F_{i}\left(h_{j_{i}}\right)\right) \cap H_{j}=\emptyset, \quad j \in J, \quad i=1,2 .
$$

Lemma 3.3. If $y \in H_{j}$ then $y$ is dominated by some $z \in \operatorname{WE}\left(F_{1}, F_{2}\right) \backslash\left(L \leqslant\left(F_{1}, F_{1}\left(h_{j_{1}}\right)\right) \cup L \leqslant\left(F_{2}, F_{2}\left(h_{j_{2}}\right)\right)\right)$.

Let $J^{\prime} \subseteq J$ be a set of indices such that $j^{\prime} \in J^{\prime}$ if $H_{j^{\prime}}$ satisfies that

$$
\left(\operatorname{WE}\left(F_{1}, F_{2}\right) \backslash\left(L \leqslant\left(F_{1}, F_{1}\left(h_{j_{1}^{\prime}}\right)\right) \cup L \leqslant\left(F_{2}, F_{2}\left(h_{j_{2}^{\prime}}\right)\right)\right)\right) \cap \operatorname{ri}(Y)=\emptyset .
$$

It is worth noting that for any $j^{\prime} \in J^{\prime}$, such that $h_{j_{i}^{\prime}} \in \mathrm{WE}\left(F_{1}, F_{2}\right)$ for $i=1,2$, the corresponding part of the chain $\mathrm{WE}\left(F_{1}, F_{2}\right)$ that joins $h_{j_{1}^{\prime}}, h_{j_{2}^{\prime}}$ must be outside of the set $Y$.

Theorem 3.2. If $\operatorname{WE}\left(F_{1}, F_{2}\right) \cap Y \neq \emptyset$, then

$$
\mathrm{WE}\left(F_{1}, F_{2} ; Y\right)=\left(\mathrm{WE}\left(F_{1}, F_{2}\right) \cap Y\right) \cup \operatorname{proj}_{Y}\left(F_{1}\right) \cup \operatorname{proj}_{Y}\left(F_{2}\right) \cup \bigcup_{j^{\prime} \in J^{\prime}} H_{j^{\prime}} .
$$

Remark 3.2. This characterization unifies, in the same sense as Theorem 3.1, the results of $[3,8,11]$ (see Example 3.4).

Proof. First of all, we have that any solution of the unconstrained problem belonging to the interior of $Y$ is a solution of the constrained problem. Indeed, if there exists $y \in \operatorname{int}(Y)$ such that $y \in \mathrm{WE}\left(F_{1}, F_{2} ; Y\right) \backslash\left(\mathrm{WE}\left(F_{1}, F_{2}\right) \cap Y\right)$ then we have that $\bigcap_{i=1}^{2} L_{<}\left(F_{i}, F_{i}(y)\right) \neq \emptyset$ and $\bigcap_{i=1}^{2} L_{<}\left(F_{i}, F_{i}(y)\right) \cap Y=\emptyset$, but this is impossible by using convexity arguments since $y \in \operatorname{int}(Y)$. Therefore, $\operatorname{WE}\left(F_{1}, F_{2} ; Y\right) \backslash\left(\mathrm{WE}\left(F_{1}, F_{2}\right) \cap Y\right) \subseteq \partial(Y)$.

Moreover, since $\operatorname{proj}_{Y}\left(F_{1}\right)$ and $\operatorname{proj}_{Y}\left(F_{2}\right)$ are the sets of points of $Y$ with the lowest objective value in $F_{1}(\cdot)$ and $F_{2}(\cdot)$, respectively, these sets belong to the nondominated solution set $\operatorname{WE}\left(F_{1}, F_{2} ; Y\right)$. Thus, we get that $\left(\operatorname{WE}\left(F_{1}, F_{2}\right) \cap Y\right) \cup$ $\left.\operatorname{}_{\operatorname{proj}_{Y}}\left(F_{1}\right) \cup \operatorname{proj}_{Y}\left(F_{2}\right)\right) \subseteq \mathrm{WE}\left(F_{1}, F_{2} ; Y\right)$. 


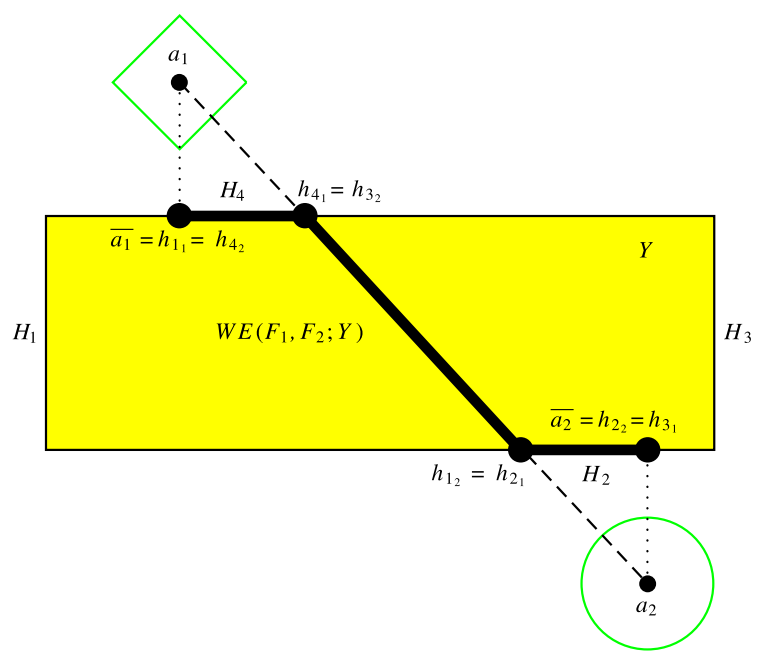

Fig. 4. Illustration of Example 3.4.

Let $j \in J \backslash J^{\prime}$ and $y \in H_{j}$. By Lemma 3.3, we have that there exists $z \in \operatorname{WE}\left(F_{1}, F_{2}\right) \backslash\left(L_{\leqslant}\left(F_{1}, F_{1}\left(h_{j_{1}}\right)\right) \cup\right.$ $\left.L \leqslant\left(F_{2}, F_{2}\left(h_{j_{2}}\right)\right)\right)$ such that $z$ dominates $y$. If $z \in Y$ then $y \notin \mathrm{WE}\left(F_{1}, F_{2} ; Y\right)$. If $z \notin Y$, we first prove that $\operatorname{ri}(\overline{z y}) \cap \partial(Y) \neq$ $\emptyset$. On the contrary, assume that $\overline{z y} \cap \partial(Y)=\{y\}$. Hence, since $\operatorname{WE}\left(F_{1}, F_{2}\right) \backslash\left(L_{\leqslant}\left(F_{1}, F_{1}\left(h_{j_{1}}\right)\right) \cup L \leqslant\left(F_{2}, F_{2}\left(h_{j_{2}}\right)\right)\right)$ is a connected set (joining $h_{j_{1}}$ and $\left.h_{j_{2}}\right)$ with empty intersection with $H_{j}$, we have that the part of $W E\left(F_{1}, F_{2}\right) \backslash\left(L_{\leqslant} \leqslant\left(F_{1}\right.\right.$, $\left.\left.F_{1}\left(h_{j_{1}}\right)\right) \cup L \leqslant\left(F_{2}, F_{2}\left(h_{j_{2}}\right)\right)\right)$ that joins $h_{j_{1}}$ and $z$ goes around either $L \leqslant\left(F_{1}, F_{1}\left(h_{j_{1}}\right)\right)$ or $L \leqslant\left(F_{2}, F_{2}\left(h_{j_{2}}\right)\right)$. Assume without loss of generality that it is around $L \leqslant\left(F_{1}, F_{1}\left(h_{j_{1}}\right)\right)$. Let $h_{z x_{1}^{*}}$ be the halfline with origin at $z$ and crossing $x_{1}^{*}$ with $x_{1}^{*} \in \mathscr{X}_{1}^{*}$. Let $z^{\prime}$ be such that $z^{\prime} \in\left(\mathrm{WE}\left(F_{1}, F_{2}\right) \backslash\left(L_{\leqslant}\left(F_{1}, F_{1}\left(h_{j_{1}}\right)\right) \cup L \leqslant\left(F_{2}, F_{2}\left(h_{j_{2}}\right)\right)\right)\right) \cap h_{z x_{1}^{*}}$. We have that $F_{1}\left(x_{1}^{*}\right)<F_{1}\left(z^{\prime}\right)$ (recall that $z^{\prime} \notin L \leqslant\left(F_{1}, F_{1}\left(h_{j_{1}}\right)\right)$ ). Moreover, since $\operatorname{WE}\left(F_{1}, F_{2}\right) \subseteq L \leqslant\left(F_{2}, F_{2}\left(x_{1}^{*}\right)\right) \cup \mathscr{X}_{1}^{*} \cup \mathscr{X}_{2}^{*}$ we have that $F_{2}(z) \leqslant F_{2}\left(x_{1}^{*}\right)$ and $F_{2}\left(z^{\prime}\right) \leqslant F_{2}\left(x_{1}^{*}\right)$. Thus, by the strict quasiconvexity of $F_{2}$ on the segment $\overline{z z^{\prime}}$ we have that $F_{2}(z)=F_{2}\left(z^{\prime}\right)=F_{2}\left(x_{1}^{*}\right)$. Hence, the part of $\mathrm{WE}\left(F_{1}, F_{2}\right)$ that joins $z$ and $z^{\prime}$ is the segment $\overline{z z^{\prime}}$ and $\overline{z z^{\prime}} \cap L \leqslant\left(F_{1}, F_{1}\left(h_{j_{1}}\right)\right) \neq$ $\emptyset$ since $x_{1}^{*} \in \overline{z z^{\prime}} \cap L \leqslant\left(F_{1}, F_{1}\left(h_{j_{1}}\right)\right)$. However, this construction contradicts the fact that the part of $\operatorname{WE}\left(F_{1}, F_{2}\right)$ that joins $z$ and $z^{\prime}$, namely $\overline{z z^{\prime}}$, has empty intersection with $L \leqslant\left(F_{1}, F_{1}\left(h_{j_{1}}\right)\right)$.

Let $w$ be such that $w \in \operatorname{ri}(\overline{y z}) \cap \partial(Y)$. By the strict quasiconvexity of the two functions, we have that $w$ dominates $y$. Hence $y \notin \mathrm{WE}\left(F_{1}, F_{2} ; Y\right)$. Therefore, $H_{j} \cap \mathrm{WE}\left(F_{1}, F_{2} ; Y\right)=\emptyset$ for all $j \in J \backslash J^{\prime}$.

Finally, since $\left(\operatorname{WE}\left(F_{1}, F_{2}\right) \backslash\left(L_{\leqslant}\left(F_{1}, F_{1}\left(h_{j_{1}^{\prime}}\right)\right) \cup L \leqslant\left(F_{2}, F_{2}\left(h_{j_{2}^{\prime}}\right)\right)\right)\right) \cap \operatorname{ri}(Y)=\emptyset \forall j^{\prime} \in J^{\prime}$ and $h_{j_{1}^{\prime}}, h_{j_{2}^{\prime}} \in \operatorname{WE}\left(F_{1}\right.$, $\left.F_{2} ; Y\right)$ using the connectedness property of the set of weakly efficient points (see [24]), we have that $H_{j^{\prime}} \subseteq$ WE $\left(F_{1}, F_{2} ; Y\right)$ and the result follows.

Example 3.4. Let $a_{1}=(4,4), a_{2}=(18,-11)$ and the feasible set, $Y$, the convex hull defined by the points $\{(0,0),(20,0)$, $(20,-7),(0,-7)\}$. For the functions $F_{1}(x)=\left\|x-a_{1}\right\|_{1}$ and $F_{2}(x)=\left\|x-a_{2}\right\|_{2}$, our goal is to determine the solution set $\operatorname{WE}\left(F_{1}, F_{2} ; Y\right)$. This set is defined by the part of the solution set of the unconstrained case (the dashed line joining $a_{1}$ and $a_{2}$ in Fig. 4) contained in $Y$ and the path on the boundary of $Y$ that connects the projections of both functions onto $Y$ with the unconstrained solution set on the boundary of $Y$ (the points $h_{4_{1}}$ and $h_{1_{2}}$ in Fig. 4). Therefore, we can see in Fig. 4, that the constrained solution set is the thick line joining the projections of both functions.

Example 3.5. Let $a=(-20,0), b=(20,0)$ be two demand points; $F_{a}(x, y)=\|(x, y)-a\|_{a}$ and $F_{b}(x, y)=\|(x, y)-b\|_{b}$ be their corresponding norms, centered at $a$ and $b$, respectively, and defined by the following unit spheres:

$$
\begin{aligned}
& \frac{4}{5} x^{2}+8 \sqrt{3} y+\frac{2}{5} y^{2}+\frac{2}{5} x(80+\sqrt{3} y)=-319, \\
& \frac{4}{5} x^{2}+8 \sqrt{3} y+\frac{2}{5} y^{2}-\frac{2}{5} x(80+\sqrt{3} y)=-319 .
\end{aligned}
$$




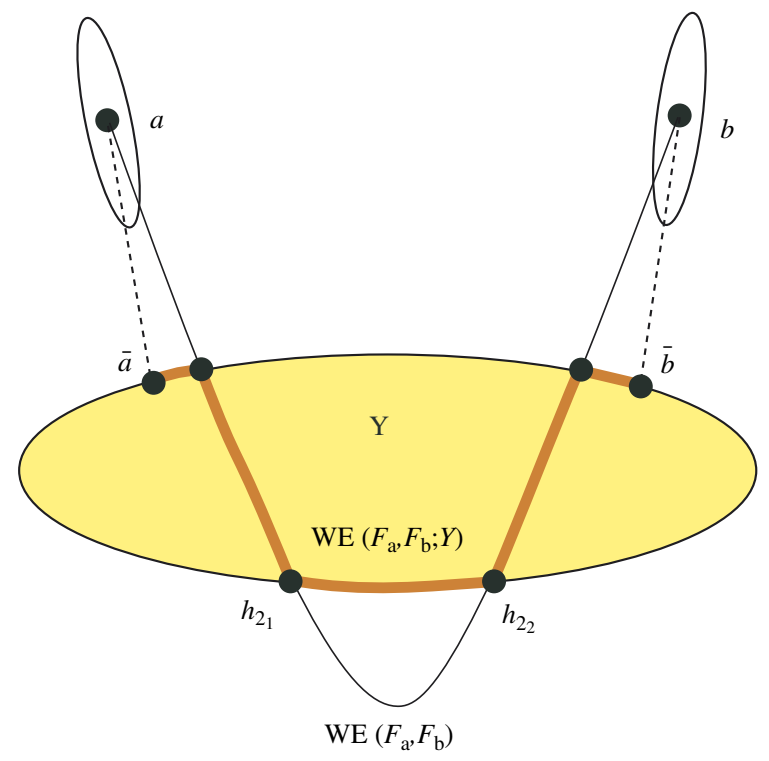

Fig. 5. Illustration of Example 3.5.

Let $Y$ be the feasible region which is the set delimited by the following ellipse:

$$
\frac{x^{2}}{400}+\frac{(y+7.5)^{2}}{25}=1
$$

Using MATHEMATICA we have computed all the unconstrained weakly efficient points for these two functions (this set is given by the tangent points of the level sets of $F_{a}$ and $F_{b}$ when the radii vary). The geometrical locus of the tangency points is defined by the following equation (the line joining $a$ and $b$ in Fig. 5):

$$
-512 \sqrt{3}+\frac{32 \sqrt{3}}{25} x^{2}-\frac{352}{5} y-\frac{16 \sqrt{3}}{25} y^{2}=0, \quad 0 \leqslant y \leqslant 20 .
$$

We have that $\bar{a}=\operatorname{proj}_{Y} F_{a}(x, y)=\{(-17.2794,-4.9822)\}$ and $\bar{b}=\operatorname{proj}_{Y} F_{b}(x, y)=\{(17.2794,-4.9822)\}$. The intersection points of the solution set in the unconstrained case with the boundary of the feasible region are

$$
\{(-16.2702,-4.5923),(-9.6629,-11.8777),(9.6629,-11.8777),(16.2702,-4.5923)\} \text {. }
$$

Applying Theorem 3.2, we have that the set $\operatorname{WE}\left(F_{a}, F_{b} ; Y\right)$ is the thick curve joining $\bar{a}$ (projection of $F_{a}$ ) and $\bar{b}$ (projection of $F_{b}$ ) in Fig. 5. Notice that this set contains the path on the boundary of $Y$ joining $h_{2_{1}}=(-9.6629,-11.8777$ ) and $h_{2_{2}}=(9.6629,-11.8777)$, since the unconstrained weakly efficient solutions that connect these two points are outside the feasible region, that is, $\left(\operatorname{WE}\left(F_{a}, F_{b}\right) \backslash\left(L \leqslant\left(F_{a}, F_{a}\left(h_{2_{1}}\right)\right) \cup L \leqslant\left(F_{b}, F_{b}\left(h_{2_{2}}\right)\right)\right)\right) \cap \operatorname{ri}(Y)=\emptyset$.

All the details of the resolution of this example using the code in the Implementation A.1 or can be sent by e-mail upon request of Appendix can be found in http://www.us.es/gpb97/curri_sevilla/index.

\section{The general multicriteria problem}

In this section, we study the three-criteria and the general $k$-criteria problems. In order to do that, we use the characterization obtained in the previous section for the two-criteria case. The following result reduces the threecriteria case to the analysis of the two criteria cases.

Theorem 4.1. If $\mathrm{WE}\left(F_{1}, F_{2}, F_{3}\right) \cap Y=\emptyset$ then

$$
\mathrm{WE}\left(F_{1}, F_{2}, F_{3} ; Y\right)=\bigcup_{i, j \in\{1,2,3\}} \mathrm{WE}\left(F_{i}, F_{j} ; Y\right) .
$$




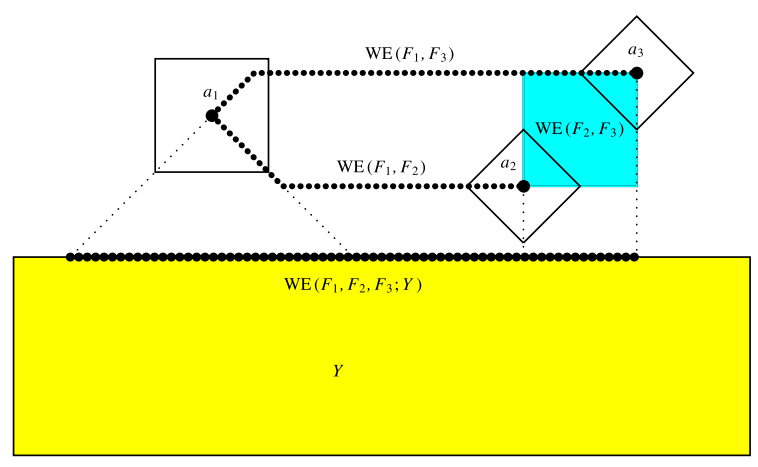

Fig. 6. Illustration of Example 4.1.

Proof. Using the same arguments that we have already used in the proof of Theorem 3.1 we obtain that $\operatorname{WE}\left(F_{1}, F_{2}\right.$, $\left.F_{3} ; Y\right) \subseteq \partial(Y)$.

On the other hand, $\bigcup_{i, j \in\{1,2,3\}} \mathrm{WE}\left(F_{i}, F_{j} ; Y\right) \subseteq \mathrm{WE}\left(F_{1}, F_{2}, F_{3} ; Y\right)$. Thus, it remains to prove that if $y \in \partial(Y) \backslash$ $\bigcup_{i, j \in\{1,2,3\}} \mathrm{WE}\left(F_{i}, F_{j} ; Y\right)$ then $y \notin \mathrm{WE}\left(F_{1}, F_{2}, F_{3} ; Y\right)$. We prove it by contradiction. Let us assume that there exists $y_{0} \in \partial(Y) \backslash \cup_{i, j \in\{1,2,3\}} \mathrm{WE}\left(F_{i}, F_{j} ; Y\right)$ such that $y_{0} \in \mathrm{WE}\left(F_{1}, F_{2}, F_{3} ; Y\right)$.

Since, $y_{0} \in Y$ and $\operatorname{WE}\left(F_{1}, F_{2}, F_{3}\right) \cap Y=\emptyset$ we have by Theorem 2.1 that $\bigcap_{i=1}^{3} L_{<}\left(F_{i}, F_{i}\left(y_{0}\right)\right) \neq \emptyset$; and thus $T_{\bigcap_{i=1}^{3} L_{<}\left(F_{i}, F_{i}\left(y_{0}\right)\right)}\left(y_{0}\right) \neq\{0\}$ (recall that the tangent cone was defined in (3)). Then by Remark 5.3.2 in [25] we have that $T_{\bigcap_{i=1}^{3} L_{<}\left(F_{i}, F_{i}\left(y_{0}\right)\right)}\left(y_{0}\right)=\bigcap_{i=1}^{3} T_{L_{<}\left(F_{i}, F_{i}\left(y_{0}\right)\right)}\left(y_{0}\right)$. The sets $T_{L_{<}\left(F_{i}, F_{i}\left(y_{0}\right)\right)}\left(y_{0}\right)$ are cones pointed at the same point $y_{0}$ for $i=1,2,3$. Therefore, there exist, $i_{0}, j_{0} \in\{1,2,3\}$ such that

$$
\bigcap_{i=1}^{3} T_{L_{<}\left(F_{i}, F_{i}\left(y_{0}\right)\right)}\left(y_{0}\right)=T_{L_{<}\left(F_{i_{0}}, F_{i_{0}}\left(y_{0}\right)\right)}\left(y_{0}\right) \cap T_{L_{<}\left(F_{j_{0}}, F_{j_{0}}\left(y_{0}\right)\right)}\left(y_{0}\right) .
$$

Moreover, since $y_{0} \notin \mathrm{WE}\left(F_{i_{0}}, F_{j_{0}} ; Y\right)$ it holds by Theorem 2.1 that $I_{i_{0} j_{0}}^{<}\left(y_{0}\right) \cap Y \neq \emptyset$ and this implies that $I_{i_{0} j_{0}}^{<}\left(y_{0}\right) \cap$ $\operatorname{ri}(Y) \neq \emptyset$. This condition permits us to write (by Remark 5.3.2 in [25]):

$$
\begin{aligned}
\emptyset & \neq \operatorname{ri}\left(T_{I_{i_{0} j_{0}}^{<}}\left(y_{0}\right) \cap Y\right. \\
& =\operatorname{ri}\left(T_{L_{<}}\left(F_{i_{0}}, F_{i_{0}}\left(y_{0}\right)\right)=\operatorname{ri}\left(T_{L_{<}\left(F_{i_{0}}, F_{i_{0}}\left(y_{0}\right)\right)}\left(y_{0}\right) \cap T_{L_{<}\left(F_{j_{0}}, F_{j_{0}}\left(y_{0}\right)\right)}\left(y_{0}\right)\right) \cap \operatorname{ri}\left(T_{Y}\left(y_{0}\right)\right)\right. \\
& =\operatorname{ri}\left(\bigcap_{i=1}^{3} T_{L_{<}\left(F_{i}, F_{i}\left(y_{0}\right)\right)}\left(y_{0}\right)\right) \cap \operatorname{ri}\left(T_{Y}\left(y_{0}\right)\right)=\operatorname{ri}\left(T_{\bigcap_{i=1}^{3} L_{<}\left(F_{i}, F_{i}\left(y_{0}\right)\right)}\left(y_{0}\right)\right) \cap \operatorname{ri}\left(T_{Y}\left(y_{0}\right)\right) .
\end{aligned}
$$

This chain proves that the set $\operatorname{ri}\left(T_{\bigcap_{i=1}^{3} L_{<}\left(F_{i}, F_{i}\left(y_{0}\right)\right)}\left(y_{0}\right)\right) \cap \operatorname{ri}\left(T_{Y}\left(y_{0}\right)\right)$ is not empty. Therefore, we choose $z \in \operatorname{ri}$ $\left(T_{\bigcap_{i=1}^{3} L_{<}\left(F_{i}, F_{i}\left(y_{0}\right)\right)}\left(y_{0}\right)\right) \cap \operatorname{ri}\left(T_{Y}\left(y_{0}\right)\right)$ and the segment $\overline{z y_{0}}$ has nonempty intersection with $\bigcap_{i=1}^{3} L_{<}\left(F_{i}, F_{i}\left(y_{0}\right)\right) \cap Y$. Hence, there exists $w \in \bigcap_{i=1}^{3} L_{<}\left(F_{i}, F_{i}\left(y_{0}\right)\right) \cap Y$ what implies that $y_{0} \notin \mathrm{WE}\left(F_{1}, F_{2}, F_{3} ; Y\right)$. This assertion contradicts the initial hypothesis and the proof is complete.

Example 4.1. We consider Example 3.1 where we have included a new point $a_{3}=(22,6.5)$ and the function $F_{3}(x)=$ $\left\|x-a_{3}\right\|_{1}$. Our goal is to determine the solution set $\operatorname{WE}\left(F_{1}, F_{2}, F_{3} ; Y\right)$. By Theorem 4.1, this set is defined by the union of the solution set for each two functions. The entire solution set is the thick line path on the boundary of $Y$ (see Fig. 6).

In order to study the general case of $k$ functions we give the following result which is a consequence of Corollary 2 in [26] and Theorem 4.1. 


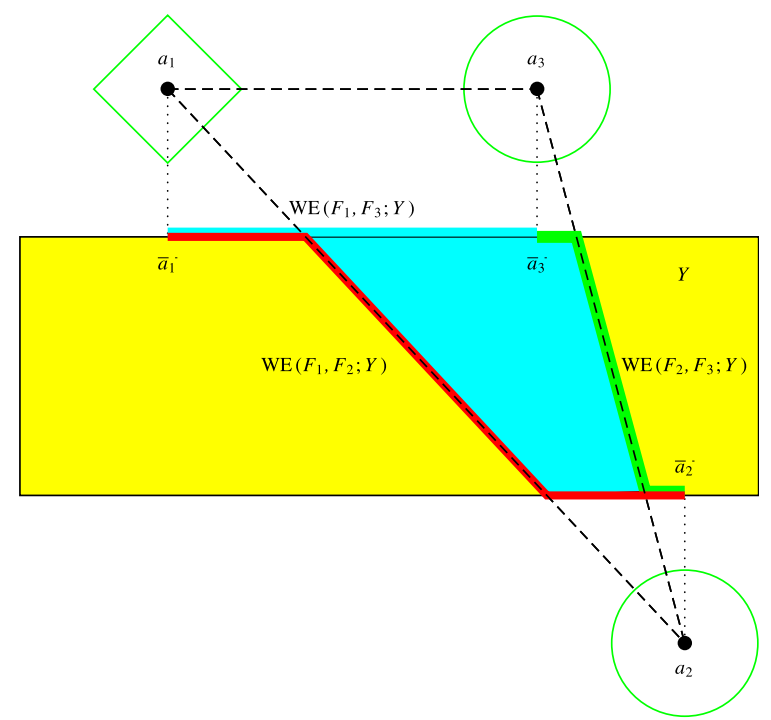

Fig. 7. Illustration of Example 4.2.

Corollary 4.1. If $\mathrm{WE}\left(F_{1}, \ldots, F_{k}\right) \cap Y=\emptyset$ then

$$
\mathrm{WE}\left(F_{1}, \ldots, F_{k} ; Y\right)=\bigcup_{i, j \in\{1, \ldots, k\}} \mathrm{WE}\left(F_{i}, F_{j} ; Y\right) .
$$

Now, we analyze the case where the intersection between the solution set in the unconstraint case and the feasible region is not empty.

Theorem 4.2. If $\mathrm{WE}\left(F_{1}, F_{2}, F_{3}\right) \cap Y \neq \emptyset$ then

$$
\mathrm{WE}\left(F_{1}, F_{2}, F_{3} ; Y\right)=\bigcup_{i, j \in\{1,2,3\}} \mathrm{WE}\left(F_{i}, F_{j} ; Y\right) \cup\left(\mathrm{WE}\left(F_{1}, F_{2}, F_{3}\right) \cap Y\right) .
$$

Proof. Using a similar argument to the one used in the proof of the Theorem 3.2, we have that $\operatorname{WE}\left(F_{1}, F_{2}, F_{3} ; Y\right) \cap$ $\operatorname{int}(Y)=\operatorname{WE}\left(F_{1}, F_{2}, F_{3}\right) \cap \operatorname{int}(Y)$.

In addition, a similar argument to the one used in Theorem 4.1 proves that $\operatorname{WE}\left(F_{1}, F_{2}, F_{3}\right) \cap \partial(Y)=\bigcup_{i, j \in\{1,2,3\}}$ $\mathrm{WE}\left(F_{i}, F_{j} ; Y\right) \cap \partial(Y)$. Hence the result follows.

Example 4.2. Consider Example 3.4 where we have included a new point $a_{3}=(14,4)$ and a new function $F_{3}(x)=$ $\left\|x-a_{3}\right\|_{2}$. Our goal is to determine the solution set $\operatorname{WE}\left(F_{1}, F_{2}, F_{3} ; Y\right)$. By Theorem 4.2, this set is defined by the unconstrained solution set included in $Y$ (the region enclosed by the dashed lines in Fig. 7) and the paths on the boundary belonging to the constrained solution set for each pair of functions.

In order to study the general case of $k$ functions, using Helly's Theorem (see [27]) we obtain the following result.

Corollary 4.2. If $\mathrm{WE}\left(F_{1}, \ldots, F_{k}\right) \cap Y \neq \emptyset$ then

$$
\mathrm{WE}\left(F_{1}, \ldots, F_{k} ; Y\right)=\bigcup_{i, j, l \in\{1, \ldots, k\}}\left[\left(\mathrm{WE}\left(F_{i}, F_{j}, F_{l}\right) \cap Y\right) \cup \bigcup_{s, t \in\{i, j, l\}} \operatorname{WE}\left(F_{s}, F_{t} ; Y\right)\right] .
$$




\section{Concluding remarks}

In this paper we have developed different geometrical characterizations of the solution set for a general constrained multicriteria location problem in two-dimensional spaces.

The approach followed in the paper unifies previous characterizations obtained for the set of weakly efficient solutions of multicriteria location problems in two-dimensional spaces. In addition, it provides a geometrical description of this kind of solution sets that allows us to actually construct it using the package MATHEMATICA.

The results presented in the paper can be extended further to the case of convex objective functions since the only technical requirement used from strictly quasiconvex functions is the behavior along rays outside the set of minimizers. In this regard, these two families of functions behave similarly and the proofs remain true.

Finally, from the characterization of the weakly efficient solutions of this paper and following a similar procedure to the one in [28], one can develop a methodology to compute the set of efficient points by checking for efficiency all the bicriteria weakly efficient chains. This issue will be investigated in a forthcoming work.

\section{Acknowledgments}

This research has been partially supported by Spanish Ministry of Education and Science Grants number BFM20012378, BFM2001-4028, BFM2004-0909 and HA2003-0121.

\section{Appendix A}

Lemma A.1. Definition 3.1 is well-stated.

Proof. Conditions (i) and (ii) in Definition 3.1 only are fulfilled by the two paths of the boundary of $Y$ connecting $\operatorname{proj}_{Y}\left(F_{1}\right)$ and $\operatorname{proj}_{Y}\left(F_{2}\right)$. In order to show that the definition is well-established, we need to prove that the condition (iii) is satisfied by only one of the two paths connecting $\operatorname{proj}_{Y}\left(F_{1}\right)$ and $\operatorname{proj}_{Y}\left(F_{2}\right)$.

In order to do that, we first prove that the set $\operatorname{encl}(C)$ is well-defined. Indeed, the sets $\mathrm{WE}\left(F_{1}, F_{2}\right)$ and $\overline{x_{1}^{*} \bar{y}_{1}} \cup \overline{\bar{y}_{1} \bar{y}_{2}} \cup$ $\overline{x_{2}^{*} \bar{y}_{2}}$ are connected. Besides, $x_{1}^{*}, x_{2}^{*} \in \mathrm{WE}\left(F_{1}, F_{2}\right)$, then $\mathrm{C}$ is a connected set. Second, we have that encl $(C) \cap \partial(Y)$ is either empty or the segment $\bar{y}_{1} \bar{y}_{2}$. Hence, by the convexity of $Y$, we have that $\operatorname{encl}(C)$ contains at most one of the two paths of the boundary of $Y$ that joins $\bar{y}_{1}$ and $\bar{y}_{2}$. In addition, if encl $(C) \cap \partial(Y)=\emptyset$, by the convexity of $Y$, we have that $\bar{y}_{1} \bar{y}_{2} \in \partial(Y)$ and $\bar{y}_{1} \bar{y}_{2} \subseteq \partial\left(\operatorname{proj}_{Y}\left(F_{1}\right), \operatorname{proj}_{Y}\left(F_{2}\right)\right)$. Thus, $\partial\left(\operatorname{proj}_{Y}\left(F_{1}\right), \operatorname{proj}_{Y}\left(F_{2}\right)\right)$ is completely defined by condition (iii). Obviously, if $Y$ is unbounded there is only one path and condition (iii) is superfluous.

Proof of Lemma 3.1. First, we have the following four assertions:

(a) Since $y_{1}, y_{2} \in Y, F_{1}\left(y_{1}\right)=F_{1}\left(y_{2}\right), F_{2}\left(y_{1}\right) \leqslant F_{2}\left(y_{2}\right)$, and using the convexity of $Y$ and the level sets we have that

$$
\overline{y_{1} y_{2}} \in I_{12}^{\leqslant}\left(y_{2}\right) \cap Y \text {. }
$$

(b) Since $y_{2} \notin \operatorname{WE}\left(F_{1}, F_{2}\right), y_{2} \in Y$ and $\operatorname{WE}\left(F_{1}, F_{2}\right) \cap Y=\emptyset$ we have that

$$
I_{12}^{<}\left(y_{2}\right) \neq \emptyset \text {. }
$$

(c) Since $y_{2} \notin \operatorname{proj}_{Y}\left(F_{1}\right)$, we have that

$$
L_{<}\left(F_{1}, F_{1}\left(y_{2}\right)\right) \cap Y \neq \emptyset .
$$

(d) Since $y_{2} \notin \operatorname{proj}_{Y}\left(F_{2}\right)$, we have that

$$
L_{<}\left(F_{2}, F_{2}\left(y_{2}\right)\right) \cap Y \neq \emptyset .
$$

Hence, using the convexity of $I_{12}^{<}\left(y_{2}\right)$ and $Y$, we have that $I_{12}^{<}\left(y_{2}\right) \cap Y \neq \emptyset$ and it implies that $y_{2} \notin W E\left(F_{1}, F_{2} ; Y\right)$. 


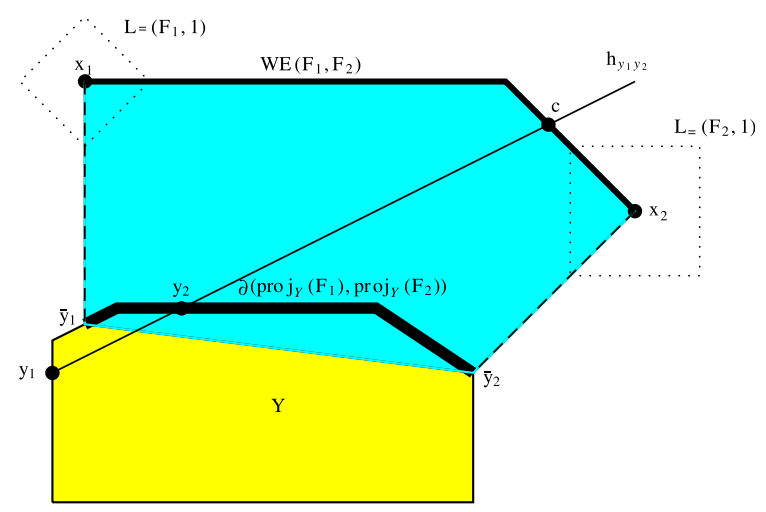

Fig. 8. $h_{y_{1} y_{2}}$ has nonempty intersection with $C \backslash \operatorname{ri}\left(\overline{\bar{y}_{1} \bar{y}_{2}}\right)$.

Now, we prove that $y_{2} \notin \partial\left(\operatorname{proj}_{Y}\left(F_{1}\right), \operatorname{proj}_{Y}\left(F_{2}\right)\right)$. Assume that $y_{2} \in \partial\left(\operatorname{proj}_{Y}\left(F_{1}\right), \operatorname{proj}_{Y}\left(F_{2}\right)\right)$. Hence, the halfline with origin at $y_{1}$ and crossing $y_{2}$, namely $h_{y_{1} y_{2}}$, has nonempty intersection with $C \backslash \operatorname{ri}\left(\overline{\bar{y}_{1} \bar{y}_{2}}\right)$, where $C$ was defined in (iii) of Definition 3.1 (see Fig. 8). Let $c \in h_{y_{1} y_{2}} \cap C \backslash \operatorname{ri}\left(\overline{\bar{y}_{1} \bar{y}_{2}}\right)$. Then, by the strict quasiconvexity of $F_{1}$ and $F_{2}$ we get that

$$
\begin{aligned}
& F_{1}\left(y_{1}\right)=F_{1}\left(y_{2}\right) \leqslant F_{1}(c), \\
& F_{2}\left(y_{1}\right) \leqslant F_{2}\left(y_{2}\right) \leqslant F_{2}(c) .
\end{aligned}
$$

Thus, since $y_{2} \notin \operatorname{WE}\left(F_{1}, F_{2}\right)$ then $c \notin \mathrm{WE}\left(F_{1}, F_{2}\right)$. Besides, since $F_{1}(c) \geqslant F_{1}\left(y_{2}\right)>F_{1}\left(\bar{y}_{1}\right)$ we have that $c \notin \overline{x_{1}^{*} \bar{y}_{1}}$. Indeed, by the strict quasiconvexity of $F_{1}$ and the fact that $\mathrm{WE}\left(F_{1}, F_{2}\right) \cap Y=\emptyset$ it holds that $F_{1}(x)<F_{1}\left(\bar{y}_{1}\right) \forall x \in$ $\overline{x_{1}^{*} \bar{y}_{1}}$. By the same arguments, since $F_{2}(c) \geqslant F_{2}\left(y_{2}\right)>F_{2}\left(\bar{y}_{2}\right)$, we have that $c \notin \overline{x_{2}^{*} \bar{y}_{2}}$. Therefore, $c \notin C \backslash \operatorname{ri}\left(\overline{\bar{y}_{1} \bar{y}_{2}}\right)$ and it contradicts the hypothesis above. Hence, $y_{2} \notin \partial\left(\operatorname{proj}_{Y}\left(F_{1}\right), \operatorname{proj}_{Y}\left(F_{2}\right)\right)$.

Remark A.1. Under the assumption that $\operatorname{proj}_{Y}\left(F_{1}\right) \cap \operatorname{proj}_{Y}\left(F_{2}\right)=\emptyset$, the hypotheses made at the beginning of Section 3.2: (1) $\operatorname{proj}_{Y}\left(F_{i}\right) \cap \operatorname{int}(Y)=\emptyset$ for $i=1,2$ and $(2) F_{1}\left(h_{j_{1}}\right)<F_{1}\left(h_{j_{2}}\right), F_{2}\left(h_{j_{2}}\right)<F_{2}\left(h_{j_{1}}\right)$ for all $j \in J$, are not restrictive.

The characterization of $\operatorname{WE}\left(F_{1}, F_{2} ; Y\right)$ when $\operatorname{proj}_{Y}\left(F_{i}\right) \cap \operatorname{int}(Y) \neq \emptyset$ for some $i=1,2$ reduces to study $\operatorname{WE}\left(F_{1}, F_{2} ; Y^{\prime}\right)$ where $\operatorname{proj}_{Y}\left(F_{i}\right) \cap \operatorname{int}\left(Y^{\prime}\right)=\emptyset$ for $i=1,2$, being $Y^{\prime}:=L \leqslant\left(F_{1}, F_{1}\left(x_{2}^{*}\right)\right) \cap L \leqslant\left(F_{2}, F_{2}\left(x_{1}^{*}\right)\right) \cap Y$ with $x_{1}^{*} \in \operatorname{argmin} x_{x \in \mathscr{X}^{*}}\left(F_{1}\right)$ $F_{2}(x)$ and $x_{2}^{*} \in \operatorname{argmin}_{x \in \mathscr{X}^{*}\left(F_{2}\right)} F_{1}(x)$. Indeed, if $\operatorname{proj}_{Y}\left(F_{i}\right) \cap \operatorname{int}(Y) \neq \emptyset$ for some $i=1,2$, then it must occur that $\mathscr{X}^{*}\left(F_{i}\right) \cap \operatorname{int}(Y) \neq \emptyset$. On the other hand, we also have that, $\operatorname{WE}\left(F_{1}, F_{2} ; Y\right) \subseteq\left(L_{\leqslant}\left(F_{1}, F_{1}\left(x_{2}^{*}\right)\right) \cap L \leqslant\left(F_{2}, F_{2}\left(x_{1}^{*}\right)\right) \cap\right.$ $Y) \cup\left(\left(\mathscr{X}^{*}\left(F_{1}\right) \cup \mathscr{X}^{*}\left(F_{2}\right)\right) \cap Y\right)$. Thus, we get that $\mathrm{WE}\left(F_{1}, F_{2} ; Y\right)=\mathrm{WE}\left(F_{1}, F_{2} ; Y^{\prime}\right) \cup\left(\left(\mathscr{X}^{*}\left(F_{1}\right) \cup \mathscr{X}^{*}\left(F_{2}\right)\right) \cap Y\right)$ where $Y^{\prime}$ satisfies that $\operatorname{proj}_{Y}\left(F_{i}\right) \cap \operatorname{int}\left(Y^{\prime}\right)=\emptyset$ for $i=1,2$. Therefore, in order to obtain a geometrical characterization of $\mathrm{WE}\left(F_{1}, F_{2} ; Y\right)$, we can assume, without loss of generality, that $\operatorname{proj}_{Y}\left(F_{i}\right) \cap \operatorname{int}(Y)=\emptyset$.

Now we prove by contradiction the second part of this remark. If $F_{1}\left(h_{j_{1}}\right)=F_{1}\left(h_{j_{2}}\right)$ two cases may occur:

(i) If $h_{j_{1}} \in \operatorname{proj}_{Y}\left(F_{1}\right)$ then $h_{j_{2}} \in \operatorname{proj}_{Y}\left(F_{1}\right)$. Thus, by the convexity of $Y$ we have that $\overline{h_{j_{1}} h_{j_{2}}} \subseteq \operatorname{proj}_{Y}\left(F_{1}\right) \subseteq \partial(Y)$. Therefore, since $H_{j} \cap\left(\operatorname{proj}_{Y}\left(F_{1}\right) \cup \operatorname{proj}_{Y}\left(F_{2}\right)\right)=\emptyset$ we have that $H_{j}=\partial(Y) \backslash \overline{h_{j_{1}} h_{j_{2}}}$. This implies that $\operatorname{proj}_{Y}\left(F_{2}\right) \subseteq$ $\overline{h_{j_{1}} h_{j_{2}}}$, that is, $\operatorname{proj}_{Y}\left(F_{1}\right) \cap \operatorname{proj}_{Y}\left(F_{2}\right) \neq \emptyset$ which is impossible.

(ii) If $h_{j_{1}} \in \mathrm{WE}\left(F_{1}, F_{2}\right) \backslash \operatorname{proj}_{Y}\left(F_{1}\right)$, since $L_{<}\left(F_{1}, F_{1}\left(h_{j_{1}}\right)\right) \cap Y \neq \emptyset, I_{12}^{<}\left(h_{j_{1}}\right)=\emptyset$ and $I_{12}^{<}\left(h_{j_{2}}\right) \cap Y=\emptyset$ then $F_{2}\left(h_{j_{1}}\right)=F_{2}\left(h_{j_{2}}\right)$. Hence, $H_{j} \cap\left(\operatorname{proj}_{Y}\left(F_{1}\right) \cup \operatorname{proj}_{Y}\left(F_{2}\right)\right) \neq \emptyset$, but it is impossible by the definition of $H_{j}$.

Proof of Lemma 3.2. In order to obtain an easy understanding we prove the result for $i=1$. By the definition of $H_{j}$, we have that $h_{j_{1}} \in \mathrm{WE}\left(F_{1}, F_{2}\right) \cup \operatorname{proj}_{Y}\left(F_{1}\right) \cup \operatorname{proj}_{Y}\left(F_{2}\right)$. If $h_{j_{1}} \in \operatorname{proj}_{Y}\left(F_{1}\right)$ by the definition of $H_{j}$ the result follows. In the following we assume, without loss of generality, that $h_{j_{i}} \notin \operatorname{proj}_{Y}\left(F_{1}\right) \cup \operatorname{proj}_{Y}\left(F_{2}\right)$ for $i=1,2$. If $h_{j_{1}} \in \operatorname{WE}\left(F_{1}, F_{2}\right)$, we distinguish two cases: (1) $H_{j} \neq \operatorname{ri}\left(\overline{h_{j_{1}} h_{j_{2}}}\right)$ and (2) $H_{j}=\mathrm{ri}\left(\overline{h_{j_{1}} h_{j_{2}}}\right)$, we prove the result in both cases by contradiction.

If $H_{j} \neq \operatorname{ri}\left(\overline{h_{j_{1}} h_{j_{2}}}\right)$, suppose that there exists $z \in L_{=}\left(F_{1}, F_{1}\left(h_{j_{1}}\right)\right) \cap H_{j}$ (observe that $z \neq h_{j_{i}}$ for $i=1$, 2 because $\left.h_{j_{i}} \notin H_{j}\right)$. Let $\bar{y}_{1} z$ be the segment that joins $\bar{y}_{1}$ and $z$ being $\bar{y}_{1} \in \operatorname{proj}_{Y}\left(F_{1}\right)$. Using that $z \in H_{j}$ and $\bar{y}_{1} \notin H_{j}$ we have 


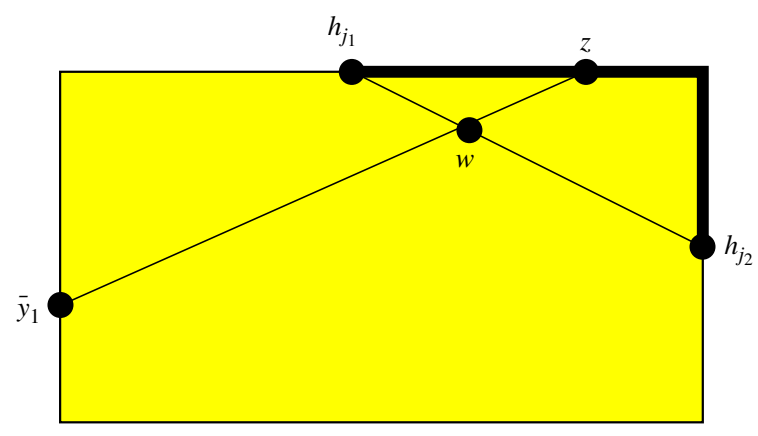

Fig. 9. $w \in \overline{\bar{y}_{1} z} \cap \overline{h_{j_{1}} h_{j_{2}}}$.

that $w$ exists, such that, $w \in \overline{y_{1} z} \cap \overline{h_{j_{1}} h_{j_{2}}}$ (see Fig. 9). (Notice that $w \neq h_{j_{i}}$ for $i=1,2$, since $\bar{y}_{1} \neq h_{j_{i}}$ and $z \neq h_{j_{i}}$ for $i=1,2$ ).

Besides, since $F_{1}(\cdot)$ is strictly quasiconvex in the line through $\bar{y}_{1}$ and $w$, and $F_{1}\left(\bar{y}_{1}\right)<F_{1}(w)$, because $w \in$ $Y \backslash \operatorname{proj}_{Y}\left(F_{1}\right)$, then $F_{1}(w)<F_{1}(z)=F_{1}\left(h_{j_{1}}\right)$. On the other hand, since $F_{2}(\cdot)$ is strictly quasiconvex in the line that joins $h_{j_{1}}$ and $h_{j_{2}}$ and $F_{2}\left(h_{j_{1}}\right)>F_{2}\left(h_{j_{2}}\right)$ then $F_{2}(w)<F_{2}\left(h_{j_{1}}\right)$. Therefore, $w$ dominates $h_{j_{1}}$. Nevertheless, this is a contradiction because $h_{j_{1}} \in \mathrm{WE}\left(F_{1}, F_{2}\right)$.

Now we study the second case, that is, $H_{j}=\operatorname{ri}\left(\overline{h_{j_{1}} h_{j_{2}}}\right)$. Assume that there exists $z \in L_{=}\left(F_{1}, F_{1}\left(h_{j_{1}}\right)\right) \cap \overline{h_{j_{1}} h_{j_{2}}}$. Since $F_{2}\left(h_{j_{2}}\right)<F_{2}\left(h_{j_{1}}\right)$, by the strict quasiconvexity of $F_{2}(\cdot)$ in the line through $h_{j_{1}}$ and $h_{j_{2}}$ we obtain that $F_{2}(z)<F_{2}\left(h_{j_{1}}\right)$. Thus, since $F_{1}\left(h_{j_{1}}\right)=F_{1}(z)$ and $h_{j_{1}} \in \mathrm{WE}\left(F_{1}, F_{2}\right)$ we have that $z \in \mathrm{WE}\left(F_{1}, F_{2}\right)$. However, it is impossible because $H_{j} \cap \mathrm{WE}\left(F_{1}, F_{2}\right)=\emptyset$.

Proof of Lemma 3.3. By Lemma 3.2, $H_{j} \cap\left(L_{\leqslant}\left(F_{1}, F_{1}\left(h_{j_{1}}\right)\right) \cup L \leqslant\left(F_{2}, F_{2}\left(h_{j_{2}}\right)\right)\right)=\emptyset$, then $F_{1}(y)>F_{1}\left(h_{j_{1}}\right)$ and $F_{2}(y)>F_{2}\left(h_{j_{2}}\right)$ for any $y \in H_{j}$. Hence, the result follows.

\section{Appendix B. The MATHEMATICA code}

The following code computes the set of weakly efficient solutions $W E(F 1, F 2 ; Y)$ provided that the feasible set $Y=\{(x, y): G(x, y) \leqslant 0\}$. To simplify the presentation we have assumed that $F 1, F 2$ and $G$ are differentiable functions. The reader may notice that this does not mean loss of generality since the polyhedral case can be also handle identifying linearity regions and applying the procedure below.

\section{B.1. Implementation of the bicriteria case}

\section{B.1.1. Implementation A.1}

\section{Input:}

$F 1\left[x_{-}, y_{-}\right]$(function $\left.F_{1}(x, y)\right)$

$F 2\left[x_{-}, y_{-}\right]$(function $F_{2}(x, y)$ )

$G\left[x_{-}, y_{-}\right]$(feasible region is given by $G(x, y) \leqslant 0$.)

2. MATHEMATICA CODE:

$\%$ (Gradient vectors)

$\left.\mathbf{D} 1\left[\mathbf{x}_{-}, \mathbf{y}_{-}\right]=\{\mathbf{D}[\mathbf{F} 1[\mathbf{x}, \mathbf{y}], \mathbf{x}], \mathbf{D}[\mathbf{F} 1[\mathbf{x}], \mathbf{y}], \mathbf{y}]\right\}$;

$\left.\mathbf{D} 2\left[\mathbf{x}_{-}, \mathbf{y}_{-}\right]=\{\mathbf{D}[\mathbf{F} 2[\mathbf{x}, \mathbf{y}], \mathbf{x}], \mathbf{D}[\mathbf{F} 2[\mathbf{x}], \mathbf{y}], \mathbf{y}]\right\}$;

$\left.\mathbf{D G}\left[\mathbf{x}_{-}, \mathbf{y}_{-}\right]=\{\mathbf{D}[\mathbf{G}[\mathbf{x}, \mathbf{y}], \mathbf{x}], \mathbf{D}[\mathbf{G}[\mathbf{x}], \mathbf{y}], \mathbf{y}]\right\}$;

$\%$ (Computation of $\operatorname{WE}\left(F_{1}, F_{2}\right)$ )

$\mathbf{W E}\left[\mathbf{x}_{-}, \mathbf{y}_{-}\right]=\operatorname{Det}[\{\mathbf{D} 1[\mathbf{x}, \mathbf{y}], \mathbf{D} 2[\mathbf{x}, \mathbf{y}]\}]$;

$\% \quad$ (Intersection points between $\operatorname{WE}\left(F_{1}, F_{2}\right)$ and $\left.\partial(Y)\right)$ 
Solve $[\{\mathbf{W E}[\mathbf{x}, \mathbf{y}]==\mathbf{0}, \mathbf{G}[\mathbf{x}, \mathbf{y}]==\mathbf{0}\},\{\mathbf{x}, \mathbf{y}\}]$

$\% \quad$ (Computation of $\operatorname{proj}_{Y}\left(F_{1}\right)$ )

$\mathbf{G 1}\left[\mathbf{x}_{-}, \mathbf{y}_{-}\right]=\operatorname{Det}[\mathrm{D1}[\mathbf{x}, \mathbf{y}], \mathbf{D G}[\mathbf{x}, \mathbf{y}]]$;

Solve $[\{\mathbf{G 1}[\mathbf{x}, \mathbf{y}]==\mathbf{0}, \mathbf{G}[\mathbf{x}, \mathbf{y}]==\mathbf{0}\},\{\mathbf{x}, \mathbf{y}\}]$;

$\% \quad$ (Computation of $\operatorname{proj}_{Y}\left(F_{2}\right)$ )

$\mathbf{G} 2\left[\mathbf{x}_{-}, \mathbf{y}_{-}\right]=\operatorname{Det}[\mathrm{D} 2[\mathbf{x}, \mathbf{y}], \mathbf{D G}[\mathbf{x}, \mathbf{y}]]$;

Solve $[\{\mathbf{G} 2[\mathbf{x}, \mathbf{y}]==\mathbf{0}, \mathbf{G}[\mathbf{x}, \mathbf{y}]==\mathbf{0}\},\{\mathbf{x}, \mathbf{y}\}]$;

$\%$ (Graphics representation of the feasible region, $\mathrm{WE}\left(F_{1}, F_{2}\right)$, and the level sets $L \leqslant\left(F_{1}, 1\right)$ and $L \leqslant\left(F_{2}, 1\right)$ )

« Graphics'ImplicitPlot'

$\mathbf{a}=\operatorname{ImplicitPlot}\left[\mathbf{F} 1[\mathbf{x}, \mathbf{y}]==\mathbf{0},\left\{\mathbf{x}, \mathbf{x}_{\mathbf{0}}, \mathbf{x}_{\mathbf{1}}\right\},\left\{\mathbf{y}, \mathbf{y}_{\mathbf{0}}, \mathbf{y}_{1}\right\}\right] ;$

$\mathbf{b}=\operatorname{ImplicitPlot}\left[\mathbf{F} 2[\mathbf{x}, \mathbf{y}]==\mathbf{0},\left\{\mathbf{x}, \mathbf{x}_{\mathbf{0}}, \mathbf{x}_{\mathbf{1}}\right\},\left\{\mathbf{y}, \mathbf{y}_{\mathbf{0}}, \mathbf{y}_{\mathbf{1}}\right\}\right]$

$\mathbf{c}=\operatorname{ImplicitPlot}\left[\mathbf{G}[\mathbf{x}, \mathbf{y}]==\mathbf{0},\left\{\mathbf{x}, \mathbf{x}_{\mathbf{0}}, \mathbf{x}_{\mathbf{1}}\right\},\left\{\mathbf{y}, \mathbf{y}_{\mathbf{0}}, \mathbf{y}_{\mathbf{1}}\right\}\right]$

$\mathbf{d}=\operatorname{ImplicitPlot}\left[\mathbf{W E}[\mathbf{x}, \mathbf{y}]==\mathbf{0},\left\{\mathbf{x}, \mathbf{x}_{\mathbf{0}}, \mathbf{x}_{\mathbf{1}}\right\},\left\{\mathbf{y}, \mathbf{y}_{\mathbf{0}}, \mathbf{y}_{\mathbf{1}}\right\}\right]$

Show $[\mathbf{a}, \mathbf{b}, \mathbf{c}, \mathbf{d}]$

\section{Appendix C. The implementation of Example 3.3}

The following MATHEMATICA code has been used to construct the level curves of the function $F_{2}$ in Example 3.3. In addition, it also computes the sequence of tangency points among the level curves of $F_{2}$ and those of $F_{1}$ when the radius $\rho$ varies. The final part draws the graphics that appear in Fig. 3.

\section{C.1. Implementation of Example 3.3}

\section{C.1.1. Implementation A. 2}

$\ll$ Graphics ${ }^{` C o l o r s ' ~} \mathbf{d 1}=\{\operatorname{Circle}[\{\mathbf{0}, \mathbf{0}\}, \mathbf{0 . 0 5 5}]\} ;$

$\mathrm{d} 2=\{\operatorname{Circle}[\{0,0\}, \mathbf{0 . 0 5 5}]\}$

$\mathrm{d} 3=\{\operatorname{Circle}[\{\mathbf{0}, 0\}, \mathbf{0 . 0 5 5}]\}$

d4 $=\{\operatorname{Circle}[\{0,0\}, 0.055]\} ;$

ps $=\{\{\mathbf{0}, \mathbf{0}\}\}$

For $[\mathbf{k}=\mathbf{1}, \mathbf{k}<\mathbf{2 0}, \mathbf{k}++$,

$\mathbf{r} 1=11\left(1-1.1^{-(5 k+1)}\right)$;

$\mathbf{r} 2=11\left(1-1.1^{-5 k}\right)$

r3 $=11\left(1-1.1^{-(5 k+2)}\right)$;

$\mathbf{r} 4=11\left(1-1.1^{-(5 k+3)}\right)$

r5 $=11\left(1-1.1^{-(5 k+4)}\right)$;

r6 $=\mathbf{1 1}\left(\mathbf{1}-\mathbf{1 . 1}^{-(5 \mathbf{k}+4)}\right)+1.2^{-(5 \mathbf{k}+4)} ;$

$\mathbf{p 1}=\left\{\left\{\mathbf{r} 1^{2} /\left(\operatorname{Sqrt}\left[\mathbf{r} 1^{2}+\mathbf{r} 2^{2}\right]\right), \mathbf{r} 2^{2} /\left(\operatorname{Sqrt}\left[\mathbf{r} 1^{2}+\mathbf{r} 2^{2}\right]\right)\right\}\right\} ;$

$\mathbf{p 2}=\left\{\left\{\mathbf{r 3}^{2} /\left(\operatorname{Sqrt}\left[\mathbf{r 3}^{2}+\mathbf{r 3}^{2}\right]\right), \mathbf{r 3}^{2} /\left(\operatorname{Sqrt}\left[\mathbf{r} 3^{2}+\mathbf{r} \mathbf{3}^{2}\right]\right)\right\}\right\} ;$

$\mathbf{p 3}=\left\{\left\{\mathbf{r} 4^{2} /\left(\operatorname{Sqrt}\left[\mathbf{r} 4^{2}+\mathbf{r 5}^{2}\right]\right), \mathbf{r 5} 5^{2} /\left(\operatorname{Sqrt}\left[\mathbf{r} 4^{2}+\mathbf{r} 5^{2}\right]\right)\right\}\right\} ;$

$\mathbf{p} 4=\left\{\left\{\mathbf{r 6}^{2} /\left(\operatorname{Sqrt}\left[\mathbf{r 6}^{2}+\mathbf{r 6}^{2}\right]\right), \mathbf{r 6} \mathbf{2}^{2} /\left(\operatorname{Sqrt}\left[\mathbf{r 6} \mathbf{2}^{2}+\mathbf{r 6}^{2}\right]\right)\right\}\right\} ;$

$\mathbf{p t}=\mathbf{U n i o n}[\mathbf{p} 1, \mathbf{p} 2, \mathbf{p} 3, \mathbf{p} 4, \mathbf{p s}]$;

$\operatorname{Print}[\mathrm{r1}$, , , , , r2, ", , , r3, ", , , r4, " , , , r5, " , , , r6];

ps $=\mathbf{p t}$;

$\mathbf{a}=\operatorname{Circle}[\{\mathbf{0}, \mathbf{0}\},\{\mathbf{r} 1, \mathbf{r} 2\}]$;

$\mathbf{b}=\operatorname{Circle}[\{\mathbf{0}, \mathbf{0}\}, \mathbf{r 3}]$;

$\mathrm{c}=\operatorname{Circle}[\{\mathbf{0}, \mathbf{0}\},\{\mathbf{r 4}, \mathbf{r 5}\}]$;

$\mathbf{d}=\operatorname{Circle}[\{\mathbf{0}, \mathbf{0}\}, \mathbf{r 6}]$;

$\mathbf{f 1}=\{\mathbf{a}\}$

h1 = Union $[\mathbf{f 1}, \mathbf{d 1}]$;

d1 = h1; 


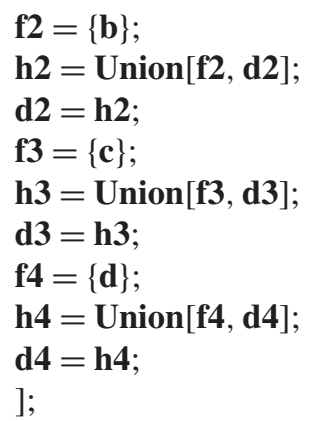

Show $[$ Graphics $[\{\{$ RGBColor $[0,0,1], \mathbf{d 1}\},\{\operatorname{RGBColor}[\mathbf{0}, \mathbf{1}, \mathbf{0}], \mathbf{d} 2\}$,

$\{$ RGBColor [1, 0, 0], d3 $\},\{$ RGBColor $[1,1,0]$, d4 $\}\}]$, AspectRatio $\rightarrow$ Automatic];

Show $[\{\{$ Graphics $[$ Line $[\{\{0,0\},\{2200,2200\}\}]]\},\{\operatorname{ListPlot}[\mathbf{p t}]\}\}]$;

Show $[\{\{$ Graphics[Line $[\{\{0,0\},\{2200,2200\}\}]]\},\{\operatorname{ListPlot}[$ pt, PlotJoined $\rightarrow$ True $]\}\}]$;

\section{References}

[1] Wendell RE, Hurter AP. Location theory, dominance and convexity. Operations Research 1973;21:314-21.

[2] Durier R. On Pareto optima, the Fermat-Weber problem and polyhedral gauges. Mathematical Programming 1990;47:65-79.

[3] Carrizosa E, Conde E, Fernández FR, Puerto J. Efficiency in Euclidean constrained location problems. Operational Research Letters 1993; 14:291-5.

[4] Carrizosa E, Conde E, Fernández FR, Puerto J. Efficiency in spatial location problems with non convex constraint. Journal of Global Optimization 1995;6:77-86.

[5] Carrizosa E, Conde E, Fernández FR, Puerto J. Multicriteria analysis with partial information about the weighting coefficients. European Journal of Operations Research 1995;81(2):291-301.

[6] Carrizosa E, Conde E, Muñoz-Márquez M, Puerto J. Simpson points in planar problems with locational constraints. The polyhedral-gauge case. Mathematics of Operations Research 1997;22(2):291-300.

[7] Carrizosa E, Conde E, Muñoz-Márquez M, Puerto J. Simpson points in planar problems with locational constraints. The round-norm case. Mathematics of Operations Research 1997;22(2):276-90.

[8] Carrizosa E, Plastria F. A characterization of efficient points in constrained location with regional demand. Operations Research Letters 1996;19(3):129-34.

[9] Durier R, Michelot C. Set of efficient points in a normed space. Journal of Mathematical Analysis and Applications 1986;117:506-28.

[10] Hansen P, Perreur J, Thisse JF. Location theory, dominance and convexity: some further results. Operations Research 1980;28(5):1241-50.

[11] Ndiaye M, Michelot C. Efficiency in constrained continuous location. European Journal of Operational Research 1998;104(2):288-98.

[12] Pelegrín R, Fernández FR. Determination of efficient points in multiple-objective location problems. Naval Research Logistics 1988; 35:697-705.

[13] Wendell RE, Hurter AP, Lowe TJ. Efficient points in location problems. AIIE Transactions 1977;9:238-46.

[14] Hamacher H, Nickel S. Multicriteria planar location problems. European Journal of Operational Research 1996;94:66-86.

[15] Puerto J, Fernández FR. A convergent approximation scheme for efficient sets of the multicriteria Weber location problem. TOP 1998; 6(2):195-204.

[16] Puerto J, Fernández FR. Multicriteria Weber location problems. Journal of the Multicriteria Decision Analysis 1999;18:268-80.

[17] Ohsawa Y. A geometrical solution for quadratic bicriteria location models. European Journal of Operational Research 1999;114:380-8.

[18] Rodríguez-Chía AM. Advances on the continuous single facility location problem. PhD Thesis, Universidad de Sevilla; 1998 [in Spanish].

[19] Rodríguez-Chía AM, Puerto J. Geometrical description of the weakly efficient solution set for multicriteria location problems. Annals of Operations Research 2002;111:181-96.

[20] Ward J. Structure of efficient sets for convex objectives. Mathematics of Operations Research 1989;14(2):249-57.

[21] Figueira J, Greco S, Ehrgott M, editors. Multiple criteria decision analysis: state of the art surveys. Berlin: Springer; 2005.

[22] Nickel S. Discretization of planar location problems. Shaker Publishers; 1995.

[23] Plastria F. Localization in single-facility location. European Journal of Operational Research 1984;18:215-9.

[24] Warburton AR. Quasiconcave vector maximization: connectedness of the sets of Pareto-optimal and weak Pareto-optimal alternatives. Journal of Optimization Theory and Applications 1983;40(4):537-57.

[25] Hiriart-Urruty JB, Lemaréchal C. Convex analysis and minimization algorithms I. Berlin: Springer; 1993.

[26] Ehrgott M, Nickel S. On the number of criteria needed to decide Pareto optimality. Mathematical Methods of Operations Research 2002; 55:329-45.

[27] Rockafellar RT. Convex analysis. Princeton, NJ: Princeton University Press; 1970.

[28] Nickel S, Puerto J, Rodríguez-Chía AM, Weissler A. Planar ordered median problems. Journal of Optimization Theory and Applications 2005;126:657-83.

[29] Carrizosa E, Plastria F. A geometrical characterization of weakly efficient points. Journal of Optimization Theory and Applications 1996;90(1):217-23. 